\title{
Improving the Quality of Physical Education Classes Using Google Services in Higher Educational Institutions
}

\author{
Svitlana Titarenko ${ }^{1}$, Dmytro Tsys ${ }^{2, *}$, Victoriya Loboda ${ }^{3}$, Nataliya Khlus ${ }^{2}$, Iryna Buzhyna ${ }^{4}$, \\ Valentina Kurilova' ${ }^{2}$, Halyna Butenko², Nataliya Tsys' ${ }^{2}$, Anatoly Kas'yan ${ }^{2}$ \\ ${ }^{1}$ Preschool Pedagogy and Psychology Chair, Oleksandr Dovzhenko Hlukhiv National Pedagogical University, Ukraine \\ ${ }^{2}$ Theory and Methodics of Physical Education Chair, Oleksandr Dovzhenko Hlukhiv National Pedagogical University, Ukraine \\ ${ }^{3}$ Theory and Methods of Education Content Chair, Sumy Regional Institute of Postgraduate Pedagogical Education, Ukraine \\ ${ }^{4}$ Department of Family and Pedagogy and Psychology, Special South Ukrainian National Pedagogical University Named after K. D. \\ Ushynsky, Ukraine
}

Received June 14, 2020; Revised August 4, 2020; Accepted August 28, 2020

\section{Cite This Paper in the following Citation Styles}

(a): [1] Svitlana Titarenko, Dmytro Tsys, Victoriya Loboda, Nataliya Khlus, Iryna Buzhyna, Valentina Kurilova, Halyna Butenko, Nataliya Tsys, Anatoly Kas'yan, "Improving the Quality of Physical Education Classes Using Google Services in Higher Educational Institutions," Universal Journal of Educational Research, Vol. 8, No. 11, pp. 5081-5091, 2020. DOI: 10.13189/ujer.2020.081108.

(b): Svitlana Titarenko, Dmytro Tsys, Victoriya Loboda, Nataliya Khlus, Iryna Buzhyna, Valentina Kurilova, Halyna Butenko, Nataliya Tsys, Anatoly Kas'yan (2020). Improving the Quality of Physical Education Classes Using Google Services in Higher Educational Institutions. Universal Journal of Educational Research, 8(11), 5081-5091. DOI: 10.13189/ujer.2020.081108.

Copyright $\bigcirc 2020$ by authors, all rights reserved. Authors agree that this article remains permanently open access under the terms of the Creative Commons Attribution License 4.0 International License

\begin{abstract}
The article deals with the problem of using Google services in the process of conducting physical education classes in higher educational institutions. The main Google products are characterized in the article; it is proved that their competent use in the process of physical education allows increasing the level of both cognitive activity and physical readiness of student youth in the process of conducting physical education classes at the university. To obtain the necessary information, general scientific methods of the theoretical level of research were used, namely: analysis of scientific and methodological literature of the Ukrainian and foreign scientists; sociological method of scientific and pedagogical research (questionnaire); pedagogical methods (pedagogical experiment, pedagogical observation); methods of mathematical statistics for the processing of research results. 50 first-year male students were involved in the experiment. The control and experimental groups were made up of 25 students each. At the beginning of October (beginning of the experiment) and at the end of May (end of the experiment) the students of the control and
\end{abstract}

experimental groups took tests to define physical readiness indicators, namely: back strength, strength endurance (push-ups, leg sit on bar), flexibility (seated forward bend). The results of the study showed that conducting physical education classes using Google services positively influenced on the studied indicators: at the end of the experiment the increase in indicators in the experimental group was more than in the control one (back strength - by $5.07 \%$, push-ups - by $14.38 \%$, leg sit on bar - by $1.7 \%$, flexibility - seated forward bend - by $24.6 \%$ ). The need to form students' sustainable interest in physical self-improvement in order to strengthen their own health, increase the level of physical readiness in the process of conducting physical education classes using Google services has been proved.

Keywords Students, Google Service, Educational Process, Physical Education, Sport, Higher Education Institutions, Physical Readiness 


\section{Introduction}

The level of the country development is largely determined by the level of education development, which should quickly and adequately respond to the needs of the society. But due to its inherent conservatism, the implementation of cardinal reforms becomes more and more relevant. One of the important factors of the education reform is its informatization, which, taking into account the world experience becomes one of the actual scientific and practical problems.

The scientific and technological progress as well as the informatization of the 21 st century society has led to the development of information provision for all sections of the society. The rapid development of information and communication technologies (ICTs) contributed to the intensification of the communicative aspects of student learning activities. Nowadays there is a number of technologies in the network, through which communication between the participants of the educational process takes place, various problems are discussed, including the physical education of young people; intellectual and creative values are created; experience and information is exchanged [1].

Improving the state of health and physical readiness of modern youth is one of the most important tasks of educational institutions in Ukraine. Today, the effectiveness of physical education of young people remains unsatisfactory [2].

Physical education in higher educational institutions should promote health strengthening and motor skills developing. To solve these problems, it is necessary to study the level of students' physical readiness and taking into account the results as well as students' individual characteristics to conduct effective physical education classes.

Physical readiness of a person is one of the criteria of health, and in the practice of physical education of students in higher educational institutions, it is the main criterion for its effectiveness. Therefore, the methodics of organizing and conducting physical education classes in higher educational institutions requires further improvement, in particular the development and scientific justification of new ways of improving the quality of the physical education process [3]. So, there is a need to find new forms and methods of conducting classes as well as organizing the educational process.

\subsection{Analysis of Recent Researches and Publications}

The analysis of the pedagogical literature shows that the current state of this problem attracts the attention of scientists and practical teachers [4-8]. Online services as tools used in the educational process of the higher educational institutions are considered in a large number of publications, which has been increasing in recent years. The problems of using online services for students in certain fields, and the general aspects, advantages and disadvantages of using these tools in higher educational institutions are analyzed. In particular, these issues have been studied in the scientific publications of such scientists as [9-12].

Today, a lot of attention is devoted to the technical and technological provision of the higher educational institutions, which includes the use of the latest information technologies in the educational process. This, in turn, involves licensing and updating of software that requires significant financial costs. According to $\mathrm{S}$. Honcharova, the higher educational institution has three ways of introducing the latest information technologies into the educational process: 1) the acquisition of licensed stationary software; 2) the transition to the use of free stationary software; 3) the replacement of stationary software with web-analogues. Therefore, today the higher educational institutions have problems related to the choice of applications, among which there are free and paid products [7].

For the moment, Google is the most popular free online service that provides teamwork services and is actively used in the educational and administrative work of high school.

\subsection{The Aim}

The purpose of the study is to analyze the researches on the problem of using Google services in the process of physical education in higher educational institutions; to describe the main Google products, the competent use of which in the educational process will provide an opportunity to increase the level of students' physical readiness during physical education classes at the university.

The objectives of the study are to analyze and summarize the aspects and main ways of using information technologies in the field of physical culture and sports presented in the special scientific literature and Internet sources; to conduct a comparative analysis of first-year students' physical readiness while using Google services in the educational process at physical education classes.

\section{Materials and Methods}

50 first-year male students were involved in the experiment. The control and experimental groups were made up of 25 students each. The physical education training process in the control group remained unchanged according to the curriculum. Classes in the experimental group were conducted as follows: practical classes -4 hours per week during the academic year and 10 hours of lectures using Google services in the first and second semesters. At the beginning of October (beginning of the experiment) and at the end of May (end of the experiment) the students of the control and experimental groups took tests to define physical readiness indicators, namely: back 
strength, strength endurance (push-ups, leg sit on bar), flexibility (seated forward bend).

The study was carried out on the basis of Oleksandr Dovzhenko Hlukhiv National Pedagogical University at the Faculty of Technological and Professional Education.

According to the purpose and the objectives of the study, general scientific methods of research were used, namely: theoretical analysis of the Ukrainian and foreign scientific sources; sociological method of scientific and pedagogical research (questionnaire); pedagogical methods (pedagogical experiment, pedagogical observation); methods of mathematical statistics for the processing of research results.

Analysis of the Ukrainian and foreign scientific sources was used to study the current state of views of the scientific community on the problem of organizing the educational process in physical education using Google services. The survey was conducted to study the state of physical education and health recreation work with students in higher educational institutions; the students' motivation to do physical exercises at the classes and during extracurricular activities; to obtain information on the use of physical education means to improve mental and physical performance efficiency and use of Google services in the educational process.

Pedagogical observations were carried out throughout the experimental work. The objects of observation were students, their attitude to physical activity, as well as their general health state before, during and after classes; external signs of student fatigue were studied. The results of reaction of students' organisms to the proposed special physical exercises were analyzed.

The method of mathematical statistics was used to process the results obtained during the pedagogical experiment; software packages of applications "Mathematical Statistics", "Statistica 6.0" and software package of the program "SRSS" were studied, as they provided quantitative and qualitative analysis of indicators.

\section{Research Results}

Google current services provide the ability to form at physical education classes the necessary abilities for students, namely, the ability to search for information, to compare different sources, to find the required information, to use different types of media resources. To place materials on social services you need registration. So, if you use several services, there is a problem of remembering the logins and passwords. In this aspect, Google services have an advantage over other services, as under one account it is possible to access any service offered by Google (Figure 1 and Figure 2).

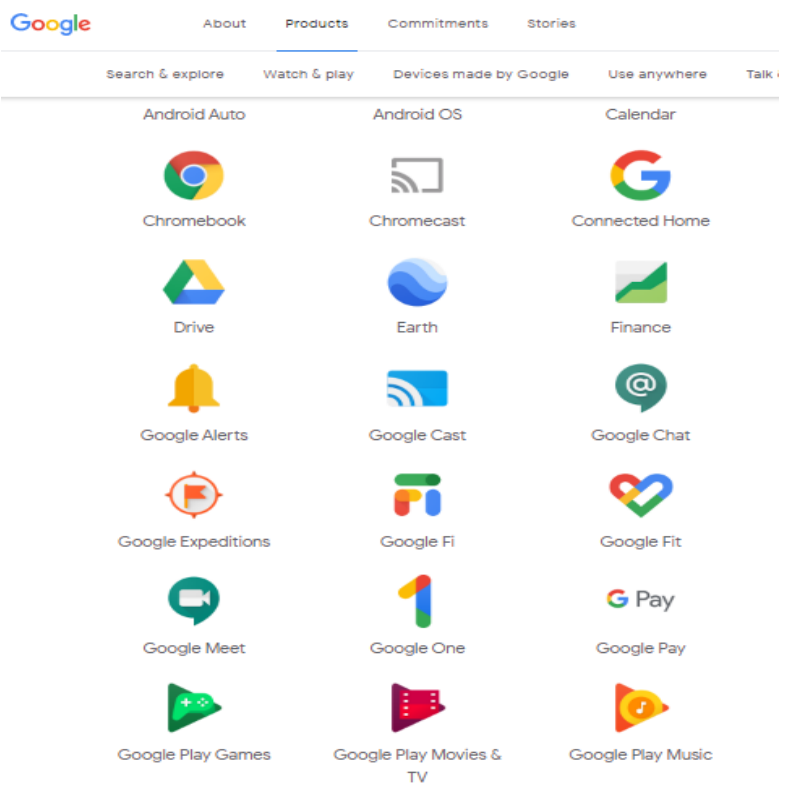

Figure 1. Google Products

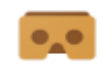

Cardboard

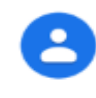

Contacts

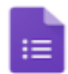

Forms

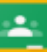

Google Classroom

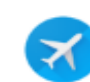

Google Flights

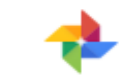

Google Photos

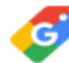

Google Shopping
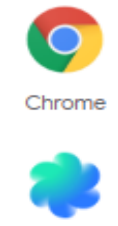

Daydream View

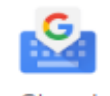

Gboard
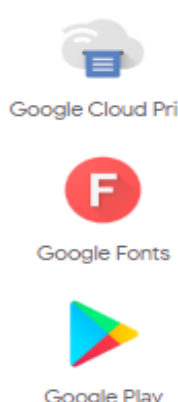

Google Play

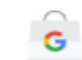

Google Store
Google Cloud Print
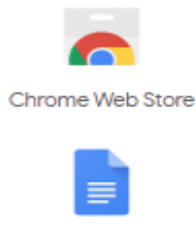

Docs

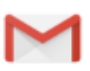

Gmail

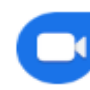

Google Duo
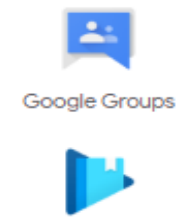

Google Play Books

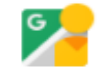

Google Street View
Figure 2. Google Products

V. Oleksyuk notes that online services are freely distributed, they are integrated, use Ukrainian language, there are those that are united by a single interface and can serve as a platform for forming the information and educational space of the higher educational institution [11]. The author emphasizes that the use of services such as Google Apps in the educational and informational environment of the higher educational institution offers significant advantages over stationary software, namely: 
reliability, high functionality; data protection; individual access to resources and services; the possibility of forming users' groups and departments; filtering unwanted content of the system or the administrator, and the user; centralized administration through an expanded set of methods and tools; a significant amount of disk space (cloud) that is provided to the user; the Ukrainian-language interface; possibility of use with mobile devices; integration with other educational facilities.

A. Shelestova emphasizes that the key aspect of Google Apps services is their constant development and improvement, which, on the one hand, can bring some inconvenience to the work of teachers and students, and on the other hand, they form their readiness for constant self-improvement [14].

The basic Google services that are used in the educational process of the higher educational institution are under consideration in the article.

Google's search engine is an online service (software-hardware suite with a Web-based interface) that provides the ability to search information on the Internet. Today, this system is the world leader in modern Internet search. This is the most powerful, reliable and high-speed search engine that delivers good search results in over 100 languages.

Google Maps (Google Maps, formerly Google Local) is a set of applications built on the basis of Google's free cartographic service and technology presented by Google. The service is a map and satellite images of the Earth planet. Now, any part of the world is available in details, and some regions can be viewed from a height of several meters. The service has an integrated business directory and a road map with directions search.

YouTube is a video hosting (a site that allows you to download and view videos in your browser, for example, using a special player), a great educational tool for teaching the techniques of exercising, rules for conducting sports games, the use of physical education equipment, etc. The educational film is an important component of the educational process, which allows both to teach and to study. This technology is indispensable for distance education as well as for online learning. Teachers have the opportunity to publish workshops and lectures recording for free, and the students can watch videos at their own pace, leaving comments and suggestions.
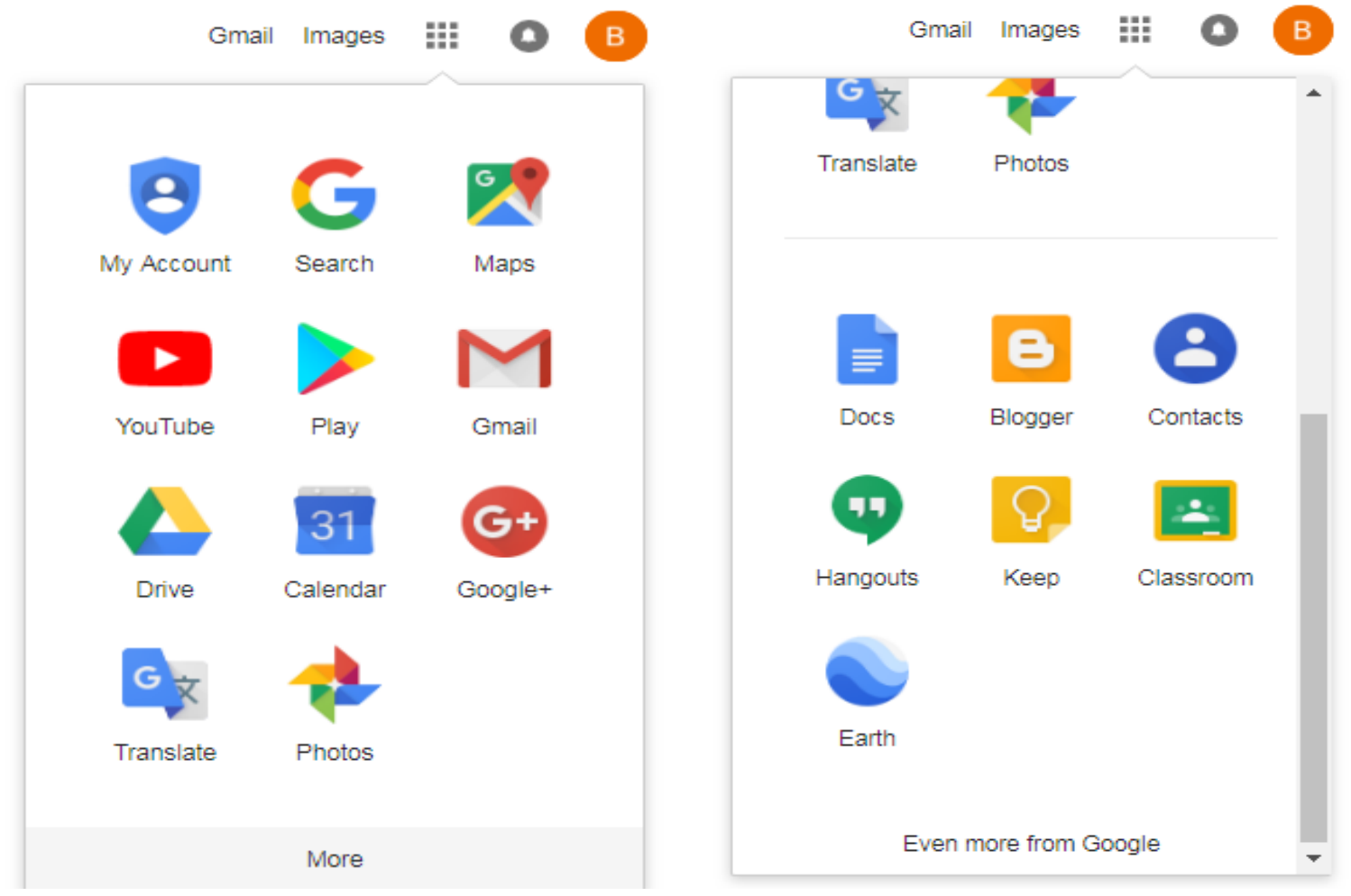

Figure 3. Google Apps 
Google + is a social network that allows the effective interaction of participants in the educational process with the help of special components: Circles, Themes, Video meetings. The entire exchange of users' materials is done in a special line (Eng. Stream) in which you can follow the updates of the circles who post messages, photos, links and videos, for example, videos, photos of sports competitions, students' participation in various conferences, projects on problems of physical education, etc.

News is a news site that is created automatically. The site has collected headlines from more than 400 news sources from all around the world: the similar articles are grouped and displayed in accordance with the personal interests of each reader. For example, news related to the use of innovative technologies in physical education, students' win in the competitions; schedule of physical culture and recreational activities, etc.

Google Translate is a system of statistical machine translation of words, texts, phrases, web pages between any pairs of languages. It can be used while translating scientific articles devoted to the study of physical education and sports problems written in a foreign language.

Blogger is a free blogging tool; it can keep track of blogging projects. Google Sites is a free web site hosting service.

The advantages of this technology include: openness of information content, efficiency of the distribution of information and access to it, independence from the individual schedule of visits by participants of the blog, interactivity - the members of the network community (students, teachers) can leave their own notes in the diary, read their colleagues' messages and publications, comment on the message and respond to comments, link messages and comments using hyperlinks. Blogs can be used for discussing and commenting on the actual problems of physical education of modern youth.

It is believed that the great communication potential and capabilities provided by Blogger and Google Sites allow you to come up with a new way of solving the following issues of organizing distance learning, such as:

- $\quad$ organization of the personal information space of a teacher and a student;

- organization of open and closed communities for the collective discussion of various physical education issues and joint work under the supervision of a moderator (teacher), which significantly expands the effectiveness of group training activities;

- creation of electronic media libraries on the actual problems of physical education and sports, which can include cataloged thematic databases of educational, reference, illustrative and other documents and data for providing address searching and free access to the network;

- providing prompt and reliable communication between the teacher and the student for solving issues aimed at forming students' readiness for physical education, receiving answers, discussing current problems and organizational issues;

- the convenience of adding, reading and searching for the necessary messages enables the deployment of constructive discussions that are an integral part of the pedagogical process;

- providing students with opportunities to share information with each other aimed at physical competence forming as well as for independent work and mutual learning;

- the use of electronic notes and lectures while training, the recommended literature in physical education issues, etc., which can be transmitted electronically, with the further formation of group consultations on various issues;

- application in the educational process of tests and surveys that allows to identify an intermediate level of knowledge or determine the students' attitude to any problem [13].

Google Mail (Gmail) is a free webmail search service that combines the best features of Google's regular email and search technology (Figure 4).

Google Apps provides each user with a Gmail account with a personalized email address. For each account, there is a storage space for mail (15GB) and built-in Google search technology that let you find the right emails as easy as finding information online with Google web search. For convenience, the emails are automatically sorted by type in Gmail. However, the size of one received message cannot exceed $25 \mathrm{MB}$. But there is an opportunity to chat in video chat, to contact a colleague or call someone without leaving the Inbox.

Investigating this problem, V. Antonov believes that Gmail, thanks to the «Important» folder, can save time if there is too much flow of incoming messages. It automatically sorts important messages and places them apart from others. Gmail uses a variety of labels to determine the importance of incoming messages, including for those who send the most emails or conversations as well as for the keywords that were present in the recently opened messages [15].

Gmail alerts to your desktop appear in pop-ups when you receive a new letter or chat message. So, even if the Gmail page is not open, you can always find out if someone wants to chat.

Describing the main advantages of Gmail, it's worth noting that unlike many free and even some paid mail services, Gmail offers an encrypted connection over SMTP /POP3/ IMAP protocols, as well as a web server-based interface through an encrypted HTTPS connection. In most other postal services, the sender's IP address is recorded (Received from). View the account access log with the IP address, access protocol (browser, mobile device, POP3, etc.) and time, as well as information about whether it works or elsewhere under this account is proposed. 


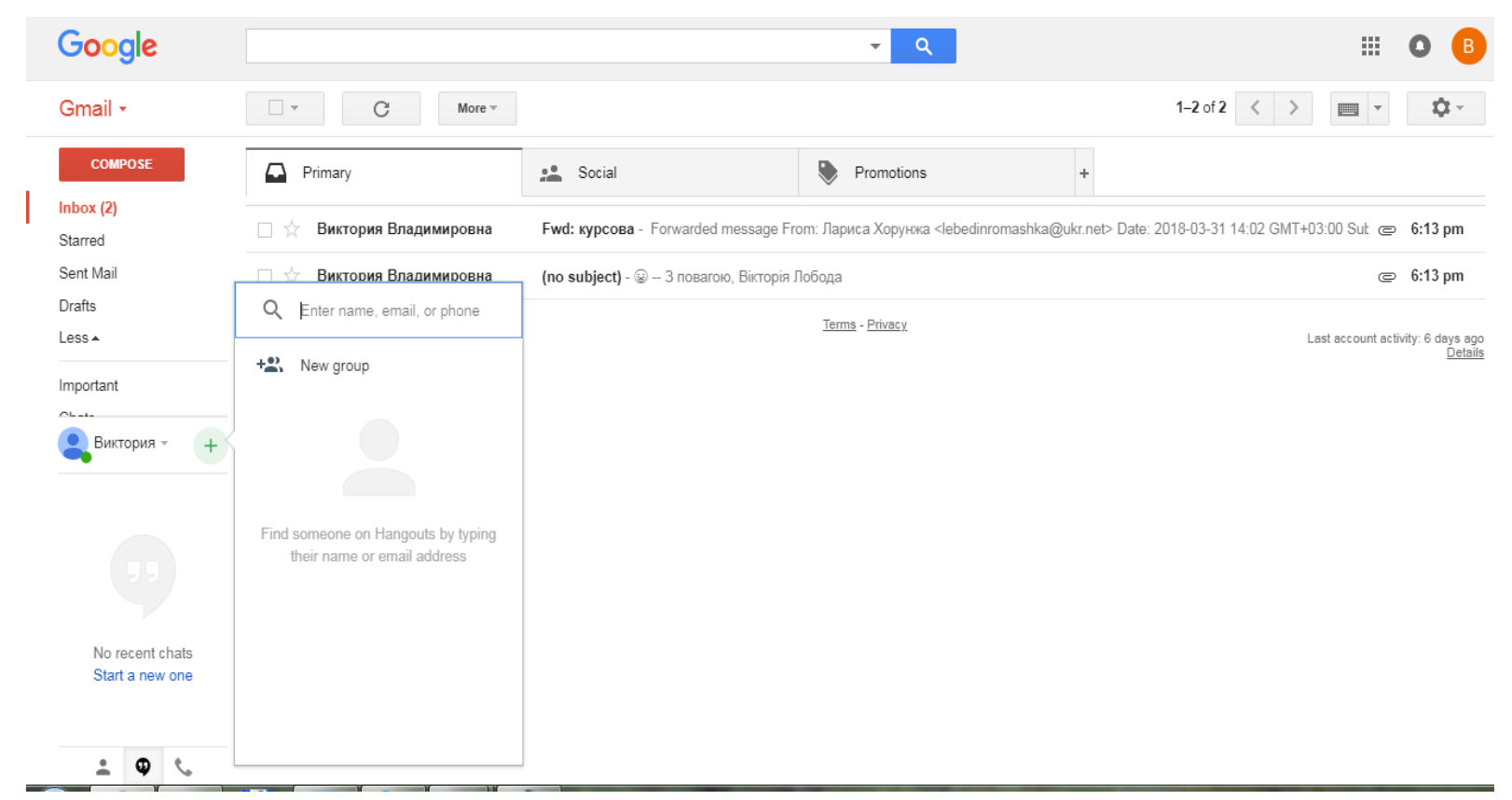

Figure 4. Gmail Interface

The analysis of special literature suggests that compared to those that have already become standard postal web services, Gmail offers a number of benefits and innovations:

1. Themes. With themes, you can customize the appearance of your Gmail account to your liking.

2. Review the discussions. The method of categorizing messages, in which Gmail tracks individual «discussions» with the initial message with a chain of responses to it (the maximum number of emails in the chain is 100). Chains are created automatically, but they cannot be created manually [5].

3. Labels. Letters are not placed into folders, but divided into categories that the user can add and modify. The effectiveness of this mechanism is higher than the more traditional with folders, as the possibility of combining different label combinations is realized. There are «standard» labels, such as inbox (inbox). With a large volume of the e-mail box you do not need to delete a letter to release the volume, it's enough to remove the inbox label (inbox) to send it to the archive.

4. Automatic save. When editing messages, it automatically scans the sheet draft several times a minute to prevent loss of data in case of power failure or other failures.

5. Developed contact list. For each interlocutor, you can add a photo, addresses and phones. Also, the substitution of addresses from the list is realized, which is displayed when typing the text in the «comma» line by the user name or his e-mail, even partially typed.

6. Hotkeys. Usage of a combination of keys to accelerate work with application. The ability to use keyboard shortcuts is rare for postal Internet services.
7. RSS support. It allows you to read letters using other RSS clients, for example, from personalized pages of search sites msn.com, yahoo.com and google.com itself, the Microsoft Deskbar. This allows you to check the mail without connecting to the web server interface.

8. Built-in spell checking. It automatically defines the language of the message and offers options for writing false words.

9. Built-in chat. Messages can be delivered not only via mail protocols but also through the Jabber protocol, allowing users to exchange instant messages using the mail server's Web server, the Google Talk application, or any other Jabber-enabled software.

10. Mail on your own domain. The ability to use the Gmail domain name for your own domain name and create a large number of mailboxes in it, each of which can take advantage of Gmail. In this case, for domains registered with non-Google partners, you will need a custom setup and-server. This feature is a part of the Google Apps service [16].

However, according to T. Valets'ka, there are certain disadvantages of the service and the limitation of its use:

1. Google privacy will automatically scan email to add contextual ads to it and protect against spam. Privacy advocates are concerned about scanning their personal, private messages, email, and consider it a security issue, although most e-mail systems use server-side scans to scan for spam. Google defends its position by referring to its use of electronic scanning to help users and states that Gmail refrains from showing ads along with potentially sensitive messages such as those that mention tragedy, disaster, or death. 
2. Technical issues. Gmail does not allow users to send or receive files and archives that contain executable files if it recognizes file extensions that are used for executable files and archives.

3. Automatic save. Gmail is unique in the sense that when editing messages by the user, auto-save is done approximately once a minute to the Drafts folder. Although this feature contributes to the reliability of services, some users complain that AutoSave is causing visible and noticeable interruptions in the input stream and is not configured under any conditions [17].

\section{Positive}

Gmail compatibility with other services. It is allowed to collect mail from other accounts. Just enter the data for authorization and specify the frequency of the charge. You can work out the opposite effect - to forward the message to another address. Using a custom filter allows you to perform selective forwarding.

Integration with other Google components is also a positive feature of the postal service. All of the web-based applications of the company have a unified interface, hot keys. There is a close exchange of data.

Google has released a number of software for various operating systems (OS) that allows you to receive messages about new mail and work with mail:

1. Gmail Notifier is a program that periodically checks your mailbox and reports new messages through the system message area (available only for Microsoft Windows and Mac OS X).

2. Google Toolbar browser extension that uses the icon to notify new messages.

3. Google Talk client for sending instant messages that supports new mail notifications.

4. Gmail Mobile Mail Client for Mobile [18].

One of the important directions of improving the efficiency of management and organization of educational process in a higher educational institution is the ability to have access to the necessary information at the right time.

Google Drive (Google Drive) and Google Docs (Google Docs) are integrated service components for storing, using, creating, modifying, and publishing any type of files.

Google Drive is a cloud-based Google repository with the ability to view online contents of many file types; it is a portable personal archive of files and folders. Disk offers $15 \mathrm{~GB}$ of free space with the ability to increase space. Google Drive allows you to store files on the Internet and on your hard drive, and access them from anywhere through the network, providing access to these resources to other users as needed. Changes made to documents are displayed on all devices that have Google Drive (mobile phone, laptop, etc.).

Simple tools are used to work with electronic documents in Google Drive: the online text editor of Google Docs, the
Google Spreadsheets - table-based online processor (Table), the Google Presentations - online presentation program (Presentation), the online graphical Google Drawings editor (Figure) and the Google Forms questionnaire form. The documents are created and processed automatically every $2-3$ seconds, each edit is saved, and you can use the function of cancellation and return of changes in the same way as in the usual local application (Figure 5).

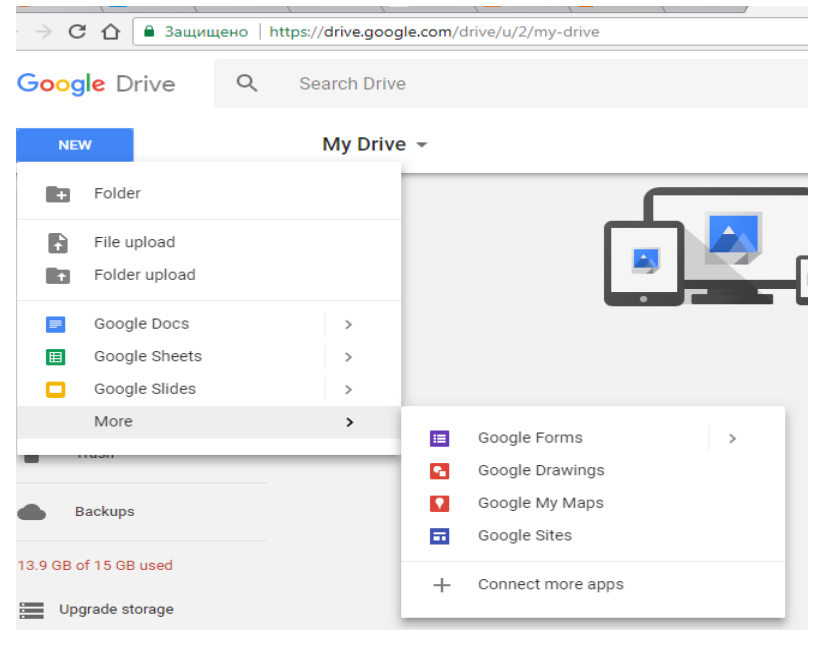

Figure 5. Google Drive Interface

The work of users takes place in real time, that is, if one of the users changes the contents of the document, all changes are immediately displayed in the windows of other users. The same file can simultaneously work up to 200 users whom the author of the document has invited to collaborate by specifying their roles: Co-author can read and edit a document, save its copy on your computer, and delete and add new collaborators; Reader can view the contents of the latest version of the document and also save it on your computer. By opening a shared document, co-authors of the provided software can process the document in real time. Everyone can add comments to the document, highlighting it in colour and adding histher name. If it is necessary, you can undo edits made by the co-authors. In the process of working with a document, you can see which of the co-authors simultaneously edits this document. A message appears above the menu bar.

Google Docs is a web-based software, a program that runs through a web browser without installing it on user's computer. Documents and spreadsheets created by the user are stored on a special Google server, or can be exported to a file. This is one of the key benefits of Google Docs as access to the entered data can be from any computer connected to the Internet (with password protected access). Working with Google Docs, you can create a new document, go back to editing the old one, or import an existing file from your computer. Google Docs is able to import files of the most common formats - RTF, DOC, TXT, HTML. In addition, to import a file, you can simply 
send it by e-mail to a special address.

In the context of our research, it is necessary to consider the benefits of using Google Docs:

1. Intelligent organization - there is no need to save documents on hard disk. Simply create a folder for them in Google Docs. In addition, if a file is lost on the computer, it is searched around the computer that is always long and not always working.

2. Auto-save - the information is automatically saved at certain intervals, there are many backups and they will not go away anywhere. In addition, if the Google server is corrupted, you can find backups on other servers.

3. Sharing is the ability to «unite» several authors in real time. This saves time while working with blog posts, documents and other texts. There is no more need to wait until it reaches the addressee by e-mail, wait until helshe looks and edits it, wait until the letter comes back. It saves not just hours, but even days of working time.

4. Correction is one of the most convenient editing tools, especially in shared access. You can see the list of changes and see who and when corrected something, compare the original and the final version, and in addition, to look at the chronology of their own changes, which may be useful for a loaded work or a careless author.

5. Bookmarks are a much underestimated feature of Google Docs. Data are very useful for long documents. If you want to move to the rest of the document you just need to insert the bookmark, and then you can easily go to this bookmark later. It's like a system of internal links. It is especially convenient to create content in this way.

6. Publications (on a blog or on Google) - with Google Docs, you can manage blogs by posting articles directly using the Publish Online tool. The only thing to do is attach the headline and blog address to the list of posts.

With the modern rhythm of life, it's hard to do without the help of special tools that help memorize the systematization of information about upcoming events and tasks. Any activity requires planning, that is why it creates programs that allow you to present data in the form of events, there are online services that allow access to data over the Internet. One of these tools for working hours is Google Calendar (Figure 6).

Google Calendar is an economical and reliable online solution for organizing educational activities and planning activities. With Google Calendar, you can easily organize and track all important events in the specified location, as well as add suggestions and send invitations to events. Google Calendar allows you to choose a convenient meeting time by sending invitations to all prospective members. In this case, the recipient of the invitation will be able to accept or reject it, which will be automatically reflected in the event description. In addition, such calendar may be open to multiple users, and changes made to it will be accessible to everyone. Google service provides the ability to run different calendars based on lifestyle and activity. For each individual calendar style, an individual access list is created.

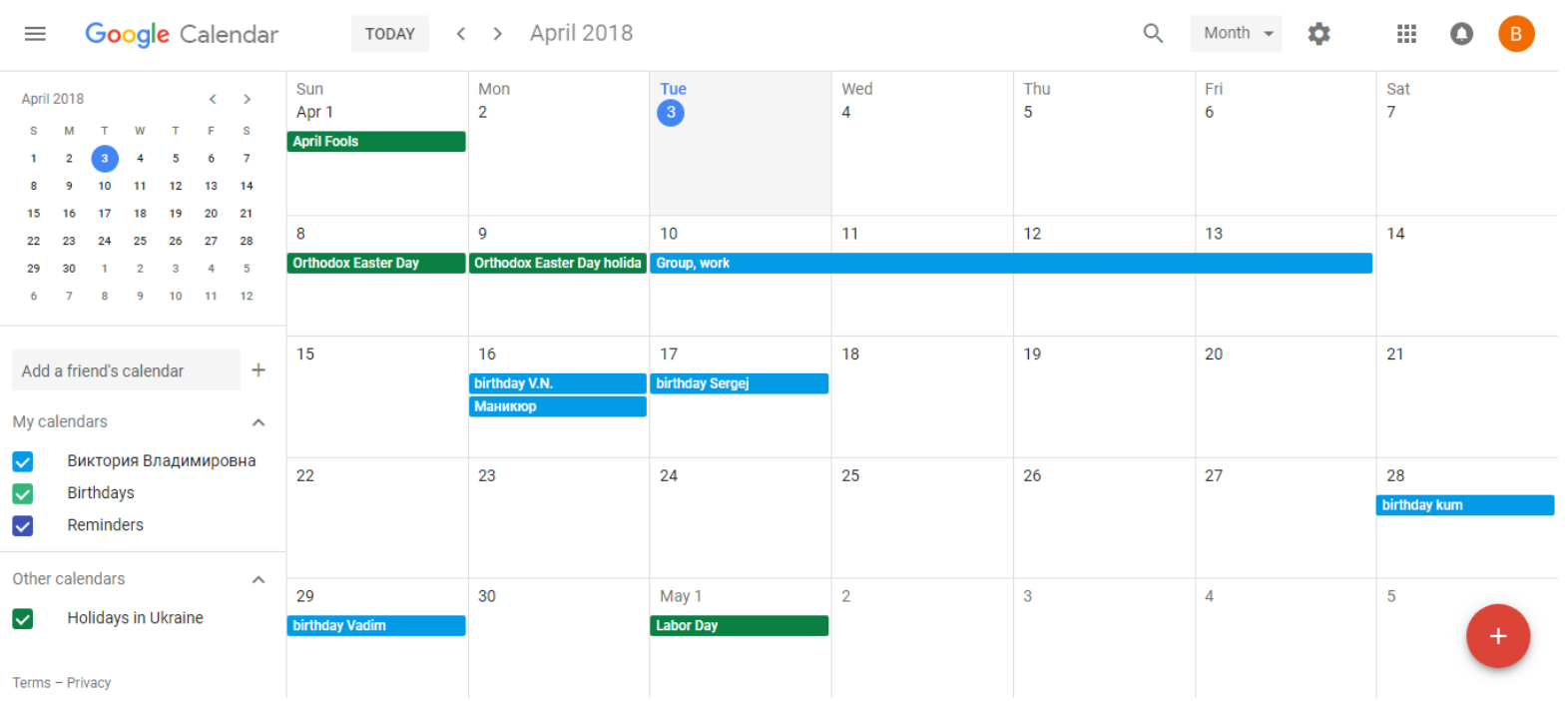

Figure 6. Google Calendar Interface 
Modern network office tools allow you to plan work jointly and create a schedule of activities that takes into account the plans of user groups. Google Calendar lets you combine the widest variety of calendars. First of all, you need to remember that calendars in this system can and should be a lot. In each calendar, it is possible to plan activities related to a certain area of activity.

With the new applications, Google services can stay working even when Internet connections are lost, and resume full-time work after it is restored. In offline mode, you can view Google Calendar events and edit documents and spreadsheets in Google Docs.

In the current circumstances, during the global pandemic COVID-19, the issue of using Google services in the educational process of higher educational institutions of various types has become relevant.

The proposed usage of Google services in the process of physical education of students positively influenced on the implementation of the goal, they contributed to improving the quality of the educational process in physical education. Let us focus on some of them. Google Maps made it possible for students to choose a place for active sports games such as frisbee, baseball, parkour, football, volleyball. They also gave students opportunity to find a location and plan the route to sports clubs, to navigate elsewhere during sports events.

Using YouTube, students had the opportunity to implement a number of tasks, including watching the proposed fragments of videos devoted to using motor skills, developing physical qualities. In addition, students shared videos that helped prepare for physical education classes. They downloaded to the smartphones necessary information to implement the given tasks in the process of physical education classes, as well as to perform independent classes, which contributed to a more effective learning of the educational material.

Google Translate helped students study the works of both Ukrainian and foreign scientists in the field of physical education and sports. Students were interested not only in the Ukrainian experience of conducting physical education classes, but also got acquainted with the organization of the physical education process in other countries of the world.

Close teacher-student communication was possible and useful with Gmail. Using this service, shared access to documents was provided, which allowed organizing distance learning. With the help of Hangouts service in electronic inbox a constant chat connection was established. In addition to chat, Hangouts allowed to create video meetings and phone calls.

Google Calendar is designed to schedule events and activities. It allowed setting the time of the meeting, set reminders for sporting events, as well as inviting other participants. With the help of Google Calendar, for the experimental group a schedule of all physical education and health recreation activities at the university as well as a schedule of section classes that students could attend of their choice were developed.

To check the effectiveness of using Google services to improve the quality of physical education of students in a higher educational institution, a pedagogical experiment was conducted.

Before forming the experimental and control groups, we previously conducted a survey of students. Based on it, both positive and negative results of the survey were selected and analyzed.

The following results of students' motivation for physical education classes using Google services were obtained: "to get pleasure from the educational process (training process)" - 37\%; "to improve health" - 50\%; "to improve general physical condition" - 40\%; "the process of training attracts" - 26\%; "other reasons (to improve physique, muscle strength, endurance, etc.)" - 32\%; "interest in classes using Google services" - 82\%

Having analyzed the obtained data, it was found out that when fulfilling the standards for physical readiness, the first-year students at the beginning of the main experiment had the results as follows: passed all standards $-36 \%$; passed part of the standards $-14 \%$; tried to pass but did not pass $-22 \%$; did not pass at all $-28 \%$. Such a negative tendency in the development of physical readiness made us search for effective means and methods, carefully plan the amount and intensity of physical activity using Google services.

In order to obtain the initial and final data and determine the feasibility of using Google services in the educational process of physical education, a level of physical readiness of first-year students was tested (Table 1).

As it can be seen in the table, the studied indicators of students at university admission were almost at the same level. However, a year later, these data of the students from the experimental group differed significantly from the data of the students from the control group. The year growth of each of the indicators of the students of the experimental group was significantly higher compared to the students of the control group. Thus, if the increase of back strength indicator among the first-year male students of the experimental group for the first year of university study using Google services was $21.22 \%$, the same indicator of the same age students from the control group was $16.15 \%$. It should be noted that the dynamics of strength endurance indicators maintain the same trend. The year growth of push-up indicator among the male students of the experimental group was $36.7 \%$, the same indicator of the male students from the control group was $22.34 \%$. The year growth of leg sit on bar indicator among the male students of the experimental group was $13 \%$, the same indicator of the male students from the control group increased only by $11.3 \%$. The most significant progress was observed in flexibility indicators: the results of sit and reach flexibility test showed that the flexibility indicator among the students of the experimental group increased by $41.3 \%$, the same indicator of the students from the control group increased only by $16.7 \%$. 
Table 1. Comparative assessment of indicators of physical readiness of students of control $(\mathrm{n} 1=25)$ and experimental $(\mathrm{n} 2=25)$ groups

\begin{tabular}{|c|c|c|c|c|}
\hline \multirow{2}{*}{ Group } & \multicolumn{2}{|c|}{$X \pm m$} & \multicolumn{2}{|c|}{ Difference } \\
\hline & Beginning of the Experiment & End of the Experiment & Absolute value & $\%$ \\
\hline \multicolumn{5}{|c|}{ Back Strength (kg) } \\
\hline Control Group & $99,38 \pm 0,87$ & $114,51 \pm 0,98$ & 15,3 & 16,15 \\
\hline $\begin{array}{l}\text { Experimental } \\
\text { Group }\end{array}$ & $98,61 \pm 0,91$ & $119,54 \pm 1,09$ & 20,93 & 21,22 \\
\hline \multicolumn{5}{|c|}{ Push-Ups (times) } \\
\hline Control Group & $15,26 \pm 0,26$ & $18,67 \pm 0,32$ & 3,41 & 22,34 \\
\hline $\begin{array}{l}\text { Experimental } \\
\text { Group }\end{array}$ & $16,12 \pm 0,23$ & $21,04 \pm 0,39$ & 4,92 & 36,72 \\
\hline \multicolumn{5}{|c|}{ Leg Sit on Bar (s) } \\
\hline Control Group & $6,8 \pm 0,19$ & $7,7 \pm 0,16$ & 0,9 & 11,3 \\
\hline $\begin{array}{l}\text { Experimental } \\
\text { Group }\end{array}$ & $6,9 \pm 0,29$ & $9,0 \pm 0,22$ & 2,1 & 13,0 \\
\hline \multicolumn{5}{|c|}{ Flexibility (Seated Forward Bend, cm) } \\
\hline Control Group & $12,6 \pm 0,24$ & $13,4 \pm 0,26$ & 0,4 & 16,7 \\
\hline $\begin{array}{c}\text { Experimental } \\
\text { Group }\end{array}$ & $12,6 \pm 0,24$ & $17,8 \pm 0,19$ & 5,2 & 41,3 \\
\hline
\end{tabular}

Thus, the research showed that physical education classes using Google services had a positive effect on the studied indicators.

\section{Conclusions and Prospects of Further Research}

The analysis of scientific and methodical literature revealed that the use of Google services in the educational process and directly during physical education classes contributes to a multifaceted positive impact on the student youth health.

The results of the study showed that physical education classes using Google services had a positive effect on the studied indicators: the indicators growth at the end of the experiment in the experimental group was more than in the control (back strength - by $5.07 \%$, push-ups - by $14.38 \%$, leg sit on bar - by $1.7 \%$, flexibility - seated forward bend by $24.6 \%$ ). The need to develop students' sustainable interest in physical self-improvement in order to strengthen their own health, increase the level of physical readiness in the process of physical education using Google services has been proved.

To conclude, it should be noted that the priority of the development of today's education is the introduction of modern information and communication technologies, which provide further improvement of the educational process, accessibility and efficiency of education, training of the younger generation for life in the information society. Education should be guided by the prospects for the development of the society. And this means that in the modern education it is necessary to apply the most up-to-date information technologies, in particular in the process of physical education in higher educational institutions. Creating a sound information environment is a key task on the path to the transition to an information society.

The prospect of our research is to develop alternative methodics using Google services that will allow controlling the health state of student youth and the level of physical readiness during the period of study in higher educational institutions, which will positively contribute to the motivation of physical education classes.

\section{Acknowledgments}

The authors would like to thank the reviewers for their useful comments on the article

\section{Disclosure Statement}

No author has any financial interest or received any financial benefit from this research.

\section{Conflict of Interest}

The authors state no conflict of interest.

\section{REFERENCES}

[1] Kademiya, M. Yu. (2011). Information and communication technologies in the educational process: a manual Vinnytsya, LLC «Glider», p. 220. 
[2] Sergienko V.M. (2007). Dynamics of development of endurance of students / Announcer of the Tchernihiv state pedagogical university of the name of T.G. Shevchenko. Issue 44. Series: pedagogical sciences. Physical education and sport: Collection it is Tchernihiv: CHDPU, pp. 281-283.

[3] Saluk I. A. (2010). Individualizaciya physical education of students with the different level of zdorov"ya: dis. on the receipt of sciences. degree of kand. ped. sciences: special. 13.00.02 "Theory and method of studies» [Electronic resource]. Kyiv $-20 \mathrm{p}$.

[4] Bartkiv, A. B. (1996). Internet Network: Today and Yesterday. Kyiv: Higher school, p. 246.

[5] Bykov, V. Yu. (2004). Open Educational Environment and Modern Network Tools for Open Education Systems. Access mode: /123456789/703/1/2.pdf http://enpuir.npu.edu.ua/bitstream

[6] Burov, Ye.V. (2006). Computer Networks: Textbook. Lviv: Magnolia Plus, p. 264.

[7] Honcharova, S.A. (2011). Use of online services of the Internet in the educational process of the university. International Congress on Informatics: Information Systems and Technologies: Materials of the International Scientific Congress. October 31. -3 november: 2 h Part 1. - Minsk: BSU, pp. 336-339.

[8] Tzetzis, G. (2008). The effect of different corrective feedback methods on the outcome and self-confidence of young athletes / George Tzetzis, Evandros Votsis and Thomas Kourtessis // Journal of Sports Science and Medicine.-2008.- Vol. 7.- P. 371-378.

[9] White, C. (2009). Towards a learner-based theory of distance language learning: The concept of the learner-context interface. In P. Hubbard (Ed.) Computer Assisted Language Learning: Critical Concepts in Linguistics. Volume IV: Present Trends and Future Directions in CALL. London: Routledge. pp. 97-112.
[10] Nocenko, A.E T. I. (2013). Using Google Services to Organize Student Practice. Pedagogical Education: Theory and Practice. Pedagogy. Psychology. - №. 19, pp. 67-69.

[11] Oleksyuk, V. P. (2013). Some aspects of the application of Google Apps services at a higher educational institution. Information technologies in education. - Whip 16, pp. $116-122$.

[12] Spirin, O. M. (2010). Information and communication technologies of teaching: criteria of internal quality assessment. Information technologies and teaching aids. №. 5 (19). Access mode: http://www.ime.edu-ua.net/em.html. Title from the screen.

[13] Yudin, O. M. (2012). Improving the effectiveness of monitoring of advertising campaigns in Google Adwords. Economy and region. № 2, pp. 137-139.

[14] Shelestova, A. M. (2015). Application of some free online applications as a tool for developing students' professional skills in the discipline «Marketing and Advertising in the Internet». Innovative computer technologies in higher school: mother science-practice Conf., (Lviv, November 17-19, 2015): National. University Lviv Polytechnic University, pp. 57-61.

[15] Antonov, V. M. (2005). Modern computer networks. Kyiv: «MK-Press», p 480.

[16] Bereza, A. M. (2001). The basis of the creation of information systems: teaching. Manual. Kyiv: KNEU, p. 214

[17] Myhovych, S. M. (2012). Informatization of education as the basis of its reformation. Access mode: http://www.mnau.edu.ua/files/02 02 01 10/mygovich/201 2- mygovich-ioor.pdf.

[18] Valetska, T. M. (2004). Computer Networks: Hardwareю. Kyiv: Elga, p. 218. 